\title{
Real-space mapping of tailored sheet and edge plasmons in graphene nanoresonators
}

\author{
A. Y. Nikitin ${ }^{1,2^{*}}$, P. Alonso-González ${ }^{1,3}$, S. Vélez ${ }^{1}$, S. Mastel ${ }^{1}$, A. Centeno ${ }^{4}$,

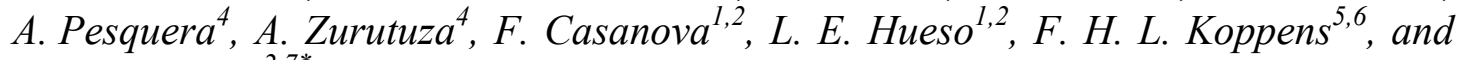 \\ R. Hillenbrand ${ }^{2,7^{*}}$
}

${ }^{1}$ CIC nanoGUNE, 20018, Donostia-San Sebastián, Spain.

${ }^{2}$ IKERBASQUE, Basque Foundation for Science, 48013 Bilbao, Spain.

${ }^{3}$ Institute of Physics, Chinese Academy of Science, Beijing 100190, China.

${ }^{4}$ Graphenea SA, 20018 Donostia-San Sebastián, Spain.

${ }^{5}$ ICFO-Institut de Ciències Fotòniques, The Barcelona Institute of Science and Technology, 08860 Castelldefels, Barcelona, Spain.

6ICREA - Institució Catalana de Recerça i Estudis Avancats, Barcelona, Spain.

${ }^{7}$ CIC NanoGUNE and UPV/EHU, E-20018, Donostia-San Sebastian, Spain

*Correspondence to: r.hillenbrand@nanogune.eu, a.nikitin@nanogune.eu

Plasmons in graphene nanoresonators have large application potential in photonics and optoelectronics, including room-temperature infrared and terahertz photodetectors, sensors, reflect-arrays or modulators ${ }^{1-7}$. Their efficient design will critically depend on the precise knowledge and control of the plasmonic modes. Here, we use near-field microscopy ${ }^{8-11}$ between $\lambda_{0}=10$ to $12 \mu \mathrm{m}$ wavelength to excite and image plasmons in tailored disk and rectangular graphene nanoresonators, and observe a rich variety of coexisting Fabry-Perot modes. Disentangling them by a theoretical analysis allows for identifying sheet and edge plasmons, the later exhibiting mode volumes as small as $10^{-8} \lambda_{0}^{3}$. By measuring the dispersion of the edge plasmons we corroborate their superior confinement compared to sheet plasmons, which among others could be applied for efficient 1D coupling of quantum emitters ${ }^{12}$. Our understanding of graphene plasmon images is a key to unprecedented in-depth analysis and verification of plasmonic functionalities in future flatland technologies. 
At infrared and terahertz frequencies, doped graphene can support electrically tunable graphene plasmons (GPs) - electromagnetic fields coupled to charge carrier oscillations - with extremely short wavelengths and large confinement ${ }^{13-17}$. For that reason, graphene has a great potential for controlling radiation on the nanometer scale $^{18}$, which largely benefits the development of highly sensitive spectroscopy ${ }^{3}$ and detection $^{19-21}$ applications. The electromagnetic field concentration achieved by GPs can be further enhanced by fabricating nanostructures acting as Fabry-Perot resonators for GPs (for example disks or ribbons) ${ }^{1,2,6,7,22}$, favoring strong absorption in arrays of the resonators (up to $40 \%)^{7}$. Until now, localized plasmonic modes in graphene ribbons and disks have been analyzed experimentally essentially by far-field spectroscopy $y^{1,2,6,7,22}$. With this technique, however, neither the mode structure, nor the unique plasmonic edge modes are accessible. A comprehensive experimental characterization of graphene plasmonic nanoresonators and their sheet and edge modes has thus been elusive so far. On the other hand, plasmonic edge modes have been shown to propagate along sharp edges of gold films, graphene and 2D electron gases $^{11,23-28}$ and provide stronger confinement of the electromagnetic fields compared to the sheet plasmons.

Here we image and analyze the near-field structure of both plasmonic sheet and edge modes in graphene disks and rectangular nanoresonators. We employ scattering-type scanning near-field optical microscopy $(\mathrm{s}-\mathrm{SNOM})^{29}$, which to date is the only available tool for real-space imaging of the propagation and confinement characteristics of graphene plasmons ${ }^{8,9,11}$. The lack of a detailed understanding of graphene-plasmonic s-SNOM contrasts, however, has not allowed yet for a comprehensive analysis of plasmon modes in graphene nanostructures. We tackled this problem by three-dimensional numerical simulations, revealing that puzzling negative plasmonic s-SNOM contrasts ${ }^{8-10}$ are governed by a spatially varying Fanolike interference between the near field of the probing tip and the graphene plasmons. This finding lets us disentangle the rich variety of coexisting Fabry-Perot modes, allowing for identifying edge and sheet modes, as well as for separating them either spatially or spectrally.

Figure 1a introduces s-SNOM imaging of localized GPs. A metal tip is used as nearfield probe. It concentrates the incident field to a nanoscale spot at the tip apex, 
providing the necessary momentum to excite GPs in a graphene nanoresonator. Interaction of the resonating GP field with the tip modifies the tip-scattered field. Recording the magnitude of this field as a function of the tip position thus yields realspace near-field images $\left|\mathrm{E}_{\mathrm{s}}(\mathrm{x}, \mathrm{y})\right|$ (see Methods).

For identifying plasmon modes and resonances, we imaged a set of disks with a diameter $d$ increasing from 50 to $450 \mathrm{~nm}$, tailored to exhibit GP resonances at the two illumination wavelengths $\lambda_{0,1}=11.06 \mu \mathrm{m}$ and $\lambda_{0,2}=11.31 \mu \mathrm{m}$ (Fig. 1b). For both wavelengths we observe several significant near-field features: a bright ring and a dark centre for the small disks (50 to $75 \mathrm{~nm}$ ), a dark ring and a bright centre for medium sized disks (150 to $200 \mathrm{~nm}$ ) and a dark centre for disks with a diameter around $350 \mathrm{~nm}$. To better understand the experimental near-field images, we plot the near-field field signal $\left|E_{s}\right|$ at two fixed tip positions (centre and edge of the disks) as a function of $d$ (Figs. 1g,h). In the disk centre, we indeed find strong peaks around $d=$ $200 \mathrm{~nm}$, providing clear evidence of a GP resonance (Fig. 1g). A resonance behavior is also found at the edge (Fig. 1h), but the peaks emerge at much smaller disk diameters (around $d=75 \mathrm{~nm}$ ), indicating that a different plasmonic mode is excited. The peaks shift to larger disk sizes when the illumination wavelength is increased from $\lambda_{0,1}$ to $\lambda_{0,2}$, which is a typical characteristics for plasmon excitations ${ }^{1,7}$. These results show that the near-field images of the disks comprise the position-dependent excitation of different plasmonic modes, which challenges the interpretation of the observed GP patterns.

We analyze the near-field images with the help of numerical electromagnetic calculations (for details see Methods and Supplementary Section A). We approximate the illuminated tip by a dipole source above the graphene disk located on a substrate (Fig 1b). By plotting the near field below the dipole as a function of its position, we obtain simulated near-field images, $\left|E_{z}(x, y)\right|$ (Fig. 1d), which are in excellent agreement with the experimental ones, $\left|\mathrm{E}_{\mathrm{s}}(\mathrm{x}, \mathrm{y})\right|$ (Fig. 1c). A good agreement is also found between the experimental and calculated resonance peaks when the tip and the dipole, respectively, are located in the disk centre (Fig. 1g and i). When the dipole is located at the disk edges, the model yields a strong peak (e1) for small diameters (Fig. 1j), which is also clearly seen in the experiment (Fig. 1h). The higher-order 
resonances seen in the simulations (e2, e3 in Fig. 1j) do not appear in the experiment (Fig. 1h), which we attribute to the limited number of data points and signal-to-noise ratio. Having established its validity, we can apply the model in the following to reliably connect the near-field image contrasts with the various GP modes.

In order to recognize the individual GP modes, we calculated the spatial near-field distribution above selected graphene disks for different fixed dipole positions (Fig. 1f). When the dipole (position marked by a green dot) is located at the centre of one of the brightest disks $(\mathrm{d}=250 \mathrm{~nm})$, we clearly recognize a circularly-symmetric breathing mode ( $\mathrm{c} 1$ in Fig. 1f), which subsequently can be assigned to the strong resonance peak in Figs. 1g,i. In contrast, a completely different plasmon mode appears when the dipole is located at the edge of the same disk (e3, Fig. 1f): the field oscillations are strongly confined to the edge and propagate along it, obviously revealing an edge mode. Perpendicular to the edge, the fields decay by a factor of $1 / \mathrm{e}$ at a distance of $\lambda_{\mathrm{e}} / 2 \pi=30 \mathrm{~nm}=\lambda_{0} / 370$, where $\lambda_{\mathrm{e}}=190 \mathrm{~nm}$ is the wavelength of the edge plasmons. With decreasing the diameter, the number of the field oscillations decreases $(\mathrm{e} 1, \mathrm{e} 2)$. Around $\mathrm{d}=100 \mathrm{~nm}$, the edge mode transforms into the fundamental dipolar mode (e1), resulting in the resonance peak at $d=75 \mathrm{~nm}$ (Figs. $1 \mathrm{~h}, \mathrm{j}$ ) and a bright ring decorating the graphene edge, which can be seen in both experiment and simulation (Figs. 1c,d). These results provide direct evidence that the tip can efficiently excite and probe both plasmonic sheet and edge modes in graphene nanoresonators.

Surprisingly, we find in both experiment and theory that the disk edge appears darker than the substrate for all diameters $d>100$ (referred to as negative near-field contrast), although edge plasmons are excited (see e.g. e4 in Fig. 1f). Similarly, the excitation of the breathing mode (c1, Fig. 1f) yields negative contrasts for disk diameters larger than $300 \mathrm{~nm}$ (i.e. the disk centre is darker than the substrate). Considering that the tip-induced GP excitation in the nanoresonators is a resonant process, we can assume that $E_{z, \mathrm{GP}}$ is phase-shifted compared to the dipole's near field above the substrate, $E_{z, \text { sub }}$. This phase shift depends on the disk size relative to the resonant disk size, and can lead to constructive or destructive interference, yielding positive or negative near-field contrast, respectively. To corroborate the phase shift 
and interference effects, we calculated both $E_{z, \text { sub }}$ and $E_{\mathrm{z}, \mathrm{GP}}$ (see Supplementary Section A). The results obtained for both e 2 and $\mathrm{c} 1$ modes are illustrated (for clarity not quantitatively) in the complex plane (Fig. 1e). We find that small phase shifts between $E_{\mathrm{z}, \mathrm{sub}}$ and $E_{\mathrm{z}, \mathrm{GP}}$ (breathing mode c1, upper panel of Fig. 1e) increase the amplitude (length of the arrows) of the total near field above graphene, $E_{z}=E_{\mathrm{z}, \mathrm{sub}}+$ $E_{z, G P}$. Conversely, for strong phase shifts (edge mode e2, lower panel of Fig. 1e) the total near-field amplitude is reduced, which shows that interference of $E_{\mathrm{z} \text {,sub }}$ and $E_{\mathrm{z}, \mathrm{GP}}$ can make plasmon patterns to appear in negative contrast in the near-field images. This effect can be interpreted as a Fano-like resonance ${ }^{30}$, where a non-resonant scattering process $\left(\mathrm{E}_{z, \text { sub }}\right)$ interferes with a resonant scattering process $\left(\mathrm{E}_{z, \mathrm{GP}}\right)$. The Fano-like resonance can be appreciated by the asymmetric line shapes in Figs. 1g,i. Based on this novel understanding of the GP near-field contrasts, we are able to properly recognize the specific GPs modes comprising the near-field images. Particularly, our analysis explains that the edges of large graphene flakes appear dark in near field images (as experimentally observed in Fig. $1 \mathrm{~b}$ and in $^{8-10}$ ), owing to the excitation of edge modes and their destructive interference with dipole's near field (Fig.S1b in Supplementary Section A).

We further studied GP modes in graphene rectangles, constituting another canonical and most important plasmonic resonator structure ${ }^{31}$. The experimental near-field images, $\left|\mathrm{E}_{\mathrm{s}}\right|$, for three rectangles of different widths $w$ but approximately equal lengths $(400 \mathrm{~nm})$ are shown in Figs. $2 \mathrm{a}-\mathrm{c}$ (top right), all of them recorded at $\lambda_{0}=11.31 \mu \mathrm{m}$. We observe a rich variety of bright and dark features, including black dots decorating the edges, which are well reproduced by simulations (Figs. 2a-c, middle right, $\left|E_{z}\right|$ ) based on the model described above. We analyze the near-field images by calculating the near field below the dipole as a function of $\lambda_{0}$ for different dipole positions (marked by green dots and labeled A-F in Fig. 2). In the resulting spectra (Figs. 2d-f) we find several peaks close to the imaging wavelength $\left(\lambda_{0}\right.$, marked by vertical lines in Figs. 2d-f), indicating the resonant excitation of different GP modes. Because of their spectral overlap, the monochromatic near-field images exhibit complex GP interference patterns. As before in Fig. 1, the asymmetric line shape of the peaks can be explained by the Fano-like interference between the tip-induced GPs, $\mathrm{E}_{\mathrm{GP}}$, and the field below the dipole, $\mathrm{E}_{\mathrm{GP}}$ (see discussion above). 
For identifying the GP modes and for recognizing them within the near-field images, we calculated the spatial near-field distribution above the resonators that are generated by the dipole located at positions A-F. In order to isolate the modes, the calculations were performed at the individual peak wavelengths. The near-field distributions (labelled w1, s1-s3 and e1-e4 in Fig. 2) clearly reveal the typical pattern of fundamental and higher order resonator modes. Similar to the disk resonators, we find direct evidence for sheet modes (s1-s3), edge modes (e1-e4) and the fundamental waveguiding mode w1 (the numbers indicate the order of the mode).

In the near-field images $\left|E_{z}\right|$ of the large resonators (Figs. 2b,c) we recognize the edge modes as rows of black dots decorating the edges. Indeed, comparison with the edge mode patterns (near-field distributions e3 and e4 in Fig. 2a) shows that the near-field maxima and minima in e3 and e4 agree well with the black dots in $\left|E_{z}\right|$ (see dashed green lines). We explain the appearance of the black dots (near-field signal oscillations revealing a standing wave pattern) by Fabry-Perot resonances of edge modes being launched by the tip and reflected at the corners of the graphene rectangles.

The smallest low-energy GP mode we find at the termination of the thin nanoresonator shown in Fig. 2a. It corresponds to the fundamental dipolar edgeplasmon resonance (near-field distribution e1 in Fig. 2a). In the near-field images it manifests as a bowtie-like bright pattern close to the resonator termination. The length of the resonant linear dipole is given by the ribbon width and is only $\mathrm{w}=70 \mathrm{~nm}$. This results in a deep subwavelength-scale field confinement of better than $\lambda_{0} / 160$ in all three dimensions, yielding an ultra-small mode volume of the order of $10^{-8} \lambda_{0}^{3}$.

A particularly interesting near-field distribution (w1 in Fig. 2a) is found when the tipdipole is placed at the centre (D) of the thinnest rectangle (Fig. 2a, middle left). The mode homogeneously extends over the whole resonator width. We explain it by the hybridization of the opposing edge modes e3 (Fig. 2b,c), which spatially overlap when the resonator width becomes smaller than the diameter of the edge modes ${ }^{24}$. In the calculated and experimental near-field images (Fig. 2a, middle right and top right) 
we recognize this mode by the row of black dots along the centre of the rectangle. Note that the w1-mode corresponds to the fundamental waveguide mode in graphene ribbons with widths $w<\lambda_{\mathrm{p}} / 2$, which can be appreciated, for the first time, in an experimental image (Fig. 2a, top right). This sub- $\lambda_{\mathrm{p}}$ waveguiding mode in the future could be exploited for the development of extremely compact subwavelength-scale graphene-plasmonic circuits, such as plasmon interferometers, for sensing and modulator applications.

Besides GP edge modes, we also recognize sheet modes. The transversal fundamental dipolar sheet mode (near-field distribution s1 in Fig. 2a) is recognized in the nearfield images of the narrowest rectangle (Figs. 2a). Similar to the near-field images of the smallest disk resonators (Figs. 1b,d), the fundamental dipolar resonance makes the graphene edges to appear bright (positive contrast), indicating that the phase shift between the fundamental dipolar GP sheet mode s1 and the tip-substrate near-field interaction is small. With increasing resonator width we observe the evolution to the higher order sheet modes (near-field distributions s2 and s3), which in the near-field images manifest as bright fringes.

We finally aim on measuring the wavelength and dispersion of the GP edge modes in comparison to sheet modes. For that reason, we study in Fig. 3 a large graphene structure (near-field image shown in Fig. 3a), which size exceeds the GP propagation length, thus avoiding Fabry-Perot resonances and sophisticated full spectral analysis $^{28}$. We find that the edge GPs launched by the tip predominately reflect at the corner, manifesting in the near-field image as a black dot located exactly at the corner (Fig. 3a, Figs. 2a-c, and Supplementary Section B ${ }^{11}$. This reflection yields interference patterns in the near-field profiles (dashed curves in Fig. 3b, extracted along the dashed white line in Fig. 3a) similar to those of the sheet plasmons (solid curves in Fig. 3b, extracted along the solid vertical white line in Fig. 3a). Interestingly, the distance $\Delta_{\mathrm{e}}$ between the two first minima of the edge profile is shorter than the distance $\Delta_{\mathrm{s}}$ observed in the sheet profile, indicating that the wavelength of edge plasmons is shorter than that of sheet plasmons. While both $\Delta_{\mathrm{e}}$ and $\Delta_{\mathrm{s}}$ decrease for shorter illumination wavelengths $\lambda_{0}$, their ratio is rather constant for all $\lambda_{0}$ (symbols in Fig. 3c), in agreement with the quasi-static analytical 
approximation for the edge plasmon dispersion in 2D electron gas ${ }^{23}$. For quantifying the wavelength of the edge plasmons, $\lambda_{\mathrm{e}}=\lambda_{\mathrm{p}} \Delta_{\mathrm{e}} / \Delta_{\mathrm{s}}$, we measure the wavelength of the sheet plasmons, $\lambda_{\mathrm{p}}$, according to previous works ${ }^{8,9}$. To that end, we measure the distance between the second and third signal maxima of the sheet profiles, yielding $\lambda_{\mathrm{p}} / 2$ (as indicated in Fig. 3a,b). The wavelengths obtained for sheet and edge plasmons are summarized in Fig. 3f (red and blue symbols, respectively). A good agreement with theory (solid lines in Fig. 3f, calculated with the help of the mode analysis) is found for a Fermi energy $E_{F}=0.33 \mathrm{eV}$ and a relaxation time $\tau=0.1 \mathrm{ps}$, verifying that with our samples the wavelength scaling of edge plasmons equals that of sheet plasmons. On the other hand, the wavelength of edge plasmons is shorter by a factor of about 1.25 , providing a superior field confinement $\left(\sim \lambda_{\mathrm{p}} / 2.5 \pi\right.$ in all directions perpendicular to the edge) compared to sheet plasmons $\left(\sim \lambda_{\mathrm{p}} / 2 \pi\right.$, only in the vertical direction). We finally corroborate and illustrate our results by calculating the nearfield distribution above the graphene, which is generated when a dipole is placed above the corner (marked by the green dot). The near-field distribution confirms that the wavelength of edge plasmons is shorter than that of sheet plasmons (Fig. 3e). We further find an excellent agreement between the calculated (Fig. 3d, employing the model described above) and experimental (Fig. 3a) near-field images, particularly confirming that the black dot at the corner is due to interference of edge plasmons launched by the tip.

In conclusion, real-space imaging and in-depth analysis of resonant GP modes in nanopatterned graphene structures has revealed a rich variety of plasmon modes, which can be categorized as sheet and edge modes. Particularly, it enabled the observation of ultra-confined resonating edge modes with mode volumes as small as $10^{-8} \lambda_{0}^{3}$. We anticipate that real-space analysis of GPs could be of great benefit for the development and quality control of emerging graphene plasmonic technologies, particularly when novel design concepts and 2D material heterostructures have to be tested and verified. Further, we believe that the developed understanding of grapheneplasmonic near-field contrasts is broadly applicable to other 2D materials. Among others, near-field microscopy could be applied for exploring polariton edge modes in thin films of van-der-Waals crystals, plasmons in nanopatterned topological 
insulators, non-reciprocal 1D plasmons ${ }^{32,33}$ or plasmons and phonons in mid-infrared and terahertz detectors based on 2D materials and heterostructures. 


\section{Methods}

\section{Fabrication of graphene resonators.}

Monolayer graphene samples were grown on a $25 \mu \mathrm{m}$-thick copper foil $(\mathrm{Cu})$ catalyst surface within a 4" chemical vapor deposition (CVD) reactor AIXTRON BM and then transferred onto two different substrates: commercial $\mathrm{Si} / \mathrm{SiO}_{2}(300 \mathrm{~nm}-$ thick$)$ and $\mathrm{CaF}_{2}$ onto which a $5 \mathrm{~nm}$-thick- $\mathrm{SiO}_{2}$ layer was sputtered on top prior graphene transfer. Then, graphene resonators of different shapes and sizes where patterned via high resolution electron-beam lithography using a thin PMMA layer, followed by a soft Ar + milling for 5 seconds and resist removal in acetone. See also Supplementary Section C.

\section{Near-field microscopy.}

Our commercially available s-SNOM (Neaspec, Munich) is based on an atomic force microscope (AFM). The vertically oscillating tip $(\Omega=250 \mathrm{kHz}$, NCSTPt, Nanosensors, Switzerland) acts as a scattering near-field probe. The oscillation amplitude was about $100 \mathrm{~nm}$. p-polarised infrared light from a grating-tunable $\mathrm{CO}_{2}$ laser was focused via a parabolic mirror onto both the tip and sample at an angle of 60 degrees to the surface normal. The tip-scattered light of field $E_{\mathrm{s}}$ was recorded with a pseudo-heterodyne interferometer ${ }^{29}$. To suppress the background scattering from the tip shaft and sample, the detector signal was demodulated at a frequency $3 \Omega$. With this technique we recorded both the amplitude and phase of $E_{\mathrm{s}}$. All figures show the magnitude of $E_{\mathrm{s}}$.

\section{Simulation model.}

The numerical calculations were done by the finite boundary elements method (Comsol). The graphene was modeled as a homogenous 2D conducting layer with the conductivity given by the random-phase approximation ${ }^{13-17}$. The conductivity jumps abruptly to zero at the graphene edge, thus breaking the translation symmetry. We completely neglect contributions from modifications of the electronic spectrum associated with the edge (e.g. electronic edge states and electrostatic effects). This treatment is justified because of the large Fermi energy, yielding large plasmon wavelengths (hundred nanometer range) compared to the localization length of electronic edge states (less than one nanometer). The edge plasmons are thus electromagnetic modes based exclusively on collective carrier oscillations. They are 
confined to the edge, owing to the broken translation symmetry of the conducting 2D sheet. Because of their strong confinement, the edge plasmons exhibit higher momenta than sheet plasmons. Consequently, the dispersions of edge and sheet plasmons are well separated form each other.

The tip was modeled by a vertically (z-) oriented point dipole source, in order to take into account that the experimental tip (i) is an elongated pyramid oriented perpendicular (vertical) to the sample and (ii) is illuminated by p-polarized light. Note that the scattering of such a tip can be approximated by an effective dipole of vertical orientation. The scattering of a vertical dipole is proportional to the z-component of the electric field below the dipole (note that horizontal fields do not couple to the vertically oriented dipole). We thus simulated the near-field images by calculating the vertical component of the near field below the dipole as a function of the dipole position, scanning the dipole parallel to the substrate above the graphene resonators. This approach is significantly different from previous simulations, where the local density of optical states was calculated ${ }^{8}$. We take into account the phase of the near fields. We are thus able to reproduce both negative and positive contrast features observed in the experimental near-field images, which have been observed previously but not explained until now.

In the calculations of Fig. 2 we had to vary slightly the Fermi energy in the calculations $\left(E_{F}=0.30 \mathrm{eV}\right.$ in Fig. $2 \mathrm{a}, E_{F}=0.28 \mathrm{eV}$ in Fig. $2 \mathrm{~b}$ and $E_{F}=0.31 \mathrm{eV}$ in Fig. $2 c$ ) in order to obtain the best agreement with the experimental and calculated nearfield images, $\left|E_{s}\right|$ and $\left(\left|E_{z}\right|\right.$, respectively. We explain this need by an inhomogeneous spatial doping of the graphene sheet.

For calculations of the spectra in Figs. 2d-f, the dipole was placed at a fixed position above the resonator, and the near field below the dipole was calculated as a function of $\lambda_{0}$. The spatial near-field distribution of the modes in Fig. 2 were obtained at the following wavelengths $\lambda_{0}$ : Fig. 2a: e1 at $11.08 \mu \mathrm{m}$, w1 at $11.64 \mu \mathrm{m}, \mathrm{s} 1$ at $10.44 \mu \mathrm{m}$; Fig. 2b: e2 at $11.31 \mu \mathrm{m}$, e3 at $11.80 \mu \mathrm{m}$, s2 at $10.56 \mu \mathrm{m}$; Fig. 2c: e4 at $11.32 \mu \mathrm{m}$, e3 $11.92 \mu \mathrm{m}, \mathrm{s} 3$ at $11.12 \mu \mathrm{m}$. They correspond to the peak values found in the spectra of 
the GP field, obtained by calculating $E_{\mathrm{z}, \mathrm{GP}}=E_{z}-E_{\mathrm{z}, \mathrm{sub}}$. For further details see Supplementary Section A.

We note that the excitation of edge modes by the dipole source can yield plasmon fields inside the resonators, as can be seen for example in the near-field distributions e3 and e4 in Fig. 2c. One explanation for this observation could be the direct coupling of the dipole fields to sheet modes, owing the partial spatial and spectral overlap of edge and sheet modes. Another explanation could be the scattering of edge modes at the corners of the resonators, providing the necessary momentum for coupling edge and sheet modes.

\section{Acknowledgements}

The authors acknowledge support from the European Union through ERC starting grants (TERATOMO grant no. 258461, SPINTROS grant no. 257654 and CarbonLight grant no. 307806), the EuropeanCommission under theGraphene Flagship (contract no.CNECTICT-604391) and the Spanish Ministry of Economy and Competitiveness (MAT2014-53432-C5-4-R, MAT2012-36580, MAT2012-37638, RYC-2012-12281, FIS2013-47161-P and 'Severo Ochoa' Programme for Centres of Excellence R\&D grant no. SEV-2015-0522). F.K. acknowledges support from the Fundacio Cellex Barcelona, the ERC Career integration grant (294056, GRANOP), the EC project GRASP (FP7-ICT-2013-613024-GRASP) and the Government of Catalonia through the SGR grant (2014-SGR-1535).

\section{Additional information}

Correspondence and requests for materials should be addressed to A.Y.N. and R.H.

\section{Competing financial interests}

R.H. is co-founder of Neaspec $\mathrm{GmbH}$, a company producing scattering-type scanning near-field optical microscope systems such as the one used in this study. All other authors declare no competing financial interests.

\section{Authors contributions:}


A.Y.N., P.A.G. and R.H. conceived the study. S.V. patterned graphene nanoresonators. A.C. and A.P. prepared CVD graphene, A.Z., F.C., L.E.H. coordinated the fabrication. P.A.G. and S.M. performed the experiments. A.Y.N. developed theory and performed the simulations. A.Y.N., P.A.G., F.H.L.K. and R.H. analysed the data and discussed the results. A.Y.N. and R.H. wrote the manuscript with the input of P.A.G. All authors contributed to the scientific discussion and manuscript revisions. 
Figures

a

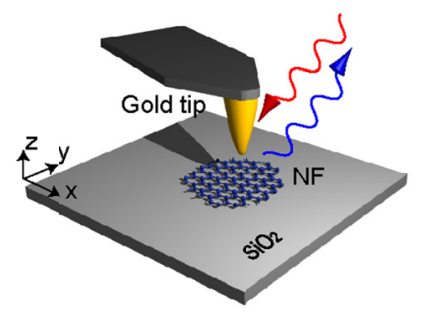

C
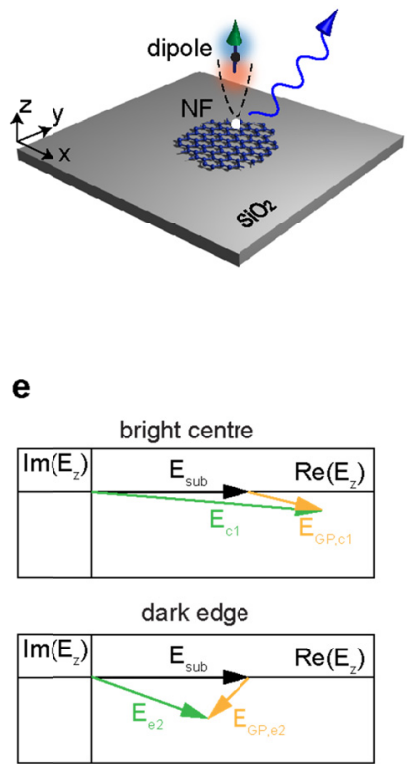

b

$\left|E_{\mathrm{s}}\right| \quad 1 \longmapsto 1.4 \quad 0.7 \square 2.5$

$d=\begin{array}{lllllllll}50 & 75 & 100 & 125 & 150 & 175 & 200 & 250 & 300\end{array}$

$\lambda_{0}=11.06 \mu \mathrm{m}$

$00: 0$

ess

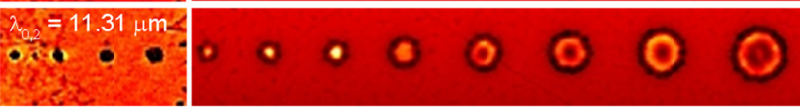

$1 \mu \mathrm{m}$

$\left|E_{z}\right| \quad 0.9 \longleftarrow 1.15 \quad 0.8 \longleftarrow 1.4$

d $\lambda_{0,1}=11.06 \mu \mathrm{m}$
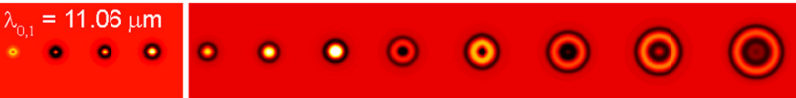

$=11.31 \mu \mathrm{m}$
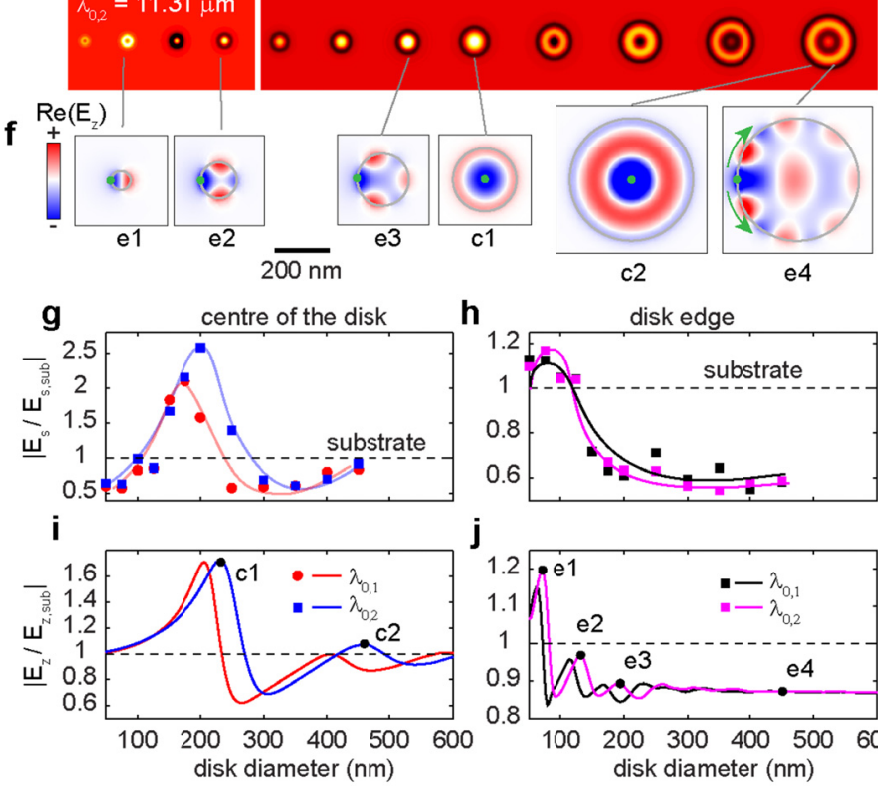

j

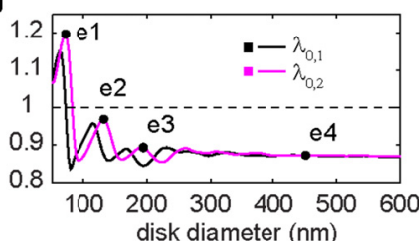

Figure 1: GP modes in graphene disk nanoresonators on $\mathrm{SiO}_{2}$ substrate. a,c, Schematics of the experiment and simulation model. b,d, Experimental and simulated near-field images at the wavelengths $\lambda_{0,1}=11.06 \mu \mathrm{m}$ and $\lambda_{0,2}=11.31 \mu \mathrm{m}$. e, Schematics illustrating the constructive, respectively destructive interference of the dipole's near field ( $\left.E_{\text {sub }}\right)$ and the GP field $\left(E_{G P}\right)$, yielding the peak $\mathrm{c} 1$ in $\mathbf{i}$, respectively peak e2 in j. f, Near-field distributions $15 \mathrm{~nm}$ above the graphene diisks induced by the dipole located at either the centre (denoted by $\mathrm{c} 1,2$ ) or the edge (denoted by e1,e2,e3 and e4). The dipole locations are marked by green dots. They correspond to the modes c1, c2 and e1-e4 marked by black dots in $\mathbf{i}$ and $\mathbf{j} . \mathbf{g}-\mathbf{j}$, Measured and calculated near-field magnitude in the disk centre and at the edge for $\lambda_{0,1}$ and $\lambda_{0,2}$. Simulations assume $E_{F}=0.33 \mathrm{eV}$ and $\tau=0.1 \mathrm{ps}$. 

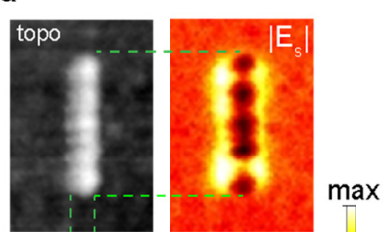

$w 1$

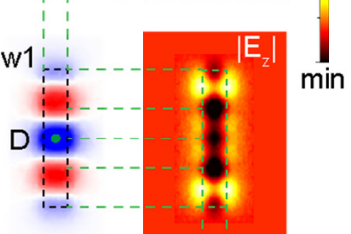

s1
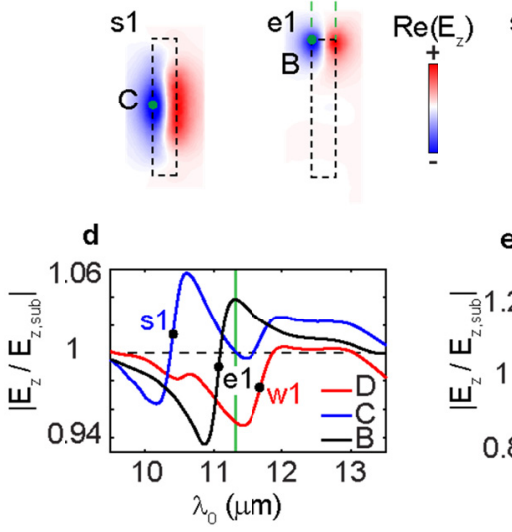

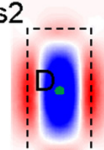

e
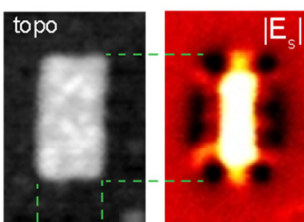

e3!

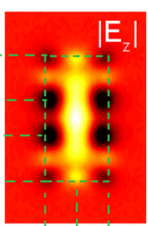

e2
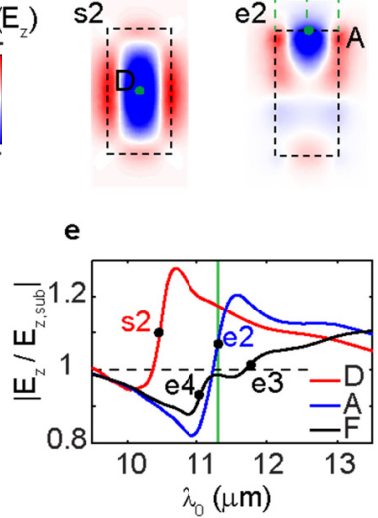
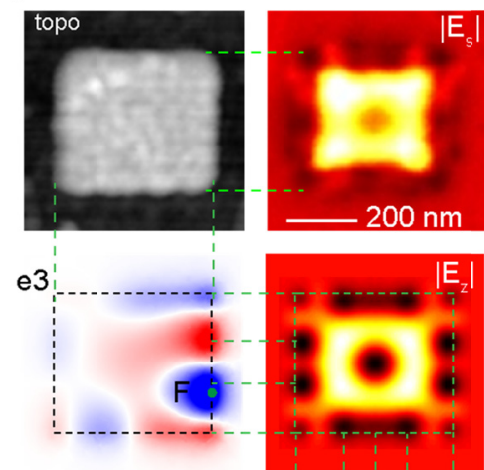

s3

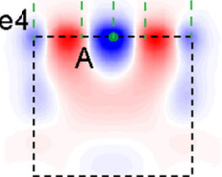

f

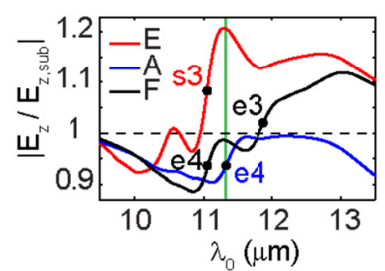

Figure 2: GP modes in rectangular graphene nanoresonators on $5 \mathrm{~nm}$-thick $\mathrm{SiO}_{2}$

film on $\mathbf{C a F}_{2}$ substrate. a-c, Topography (top left), experimental near-field image $\left(\left|\mathrm{E}_{\mathrm{s}}\right|\right.$, top right), calculated near-field image $\left(\left|\mathrm{E}_{\mathrm{z}}\right|\right.$, middle right) and calculated nearfield distributions when the dipole is placed at positions marked by green dot. Resonator sizes are $394 \times 73 \mathrm{~nm}, 360 \times 180 \mathrm{~nm}$ and $400 \times 450 \mathrm{~nm}$. Wavelength $\lambda_{0}=$ $11.31 \mu \mathrm{m}$ (marked by the vertical green line in $\mathbf{d - f}$ ). w1 marks the fundamental waveguiding mode, s1 to s3 mark the first-order to third-order transversal sheet mode. e1 to e4 mark the first-order to fourth-order edge modes. d-f, Near-field spectra calculated at the points A-F (marked by green dots in the near-field distributions). For best matching of experimental and simulated near-field images we used Fermi energies $E_{F}=0.30 \mathrm{eV}(\mathbf{a}, \mathbf{d}), E_{F}=0.28 \mathrm{eV}(\mathbf{b}, \mathbf{e})$ and $E_{F}=0.31 \mathrm{eV}(\mathbf{c}, \mathbf{f})$. Relaxation time $\tau=0.1$ ps. 
a

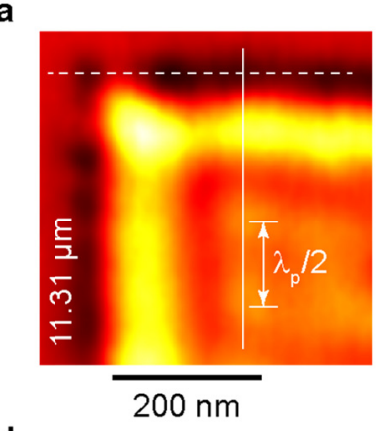

d

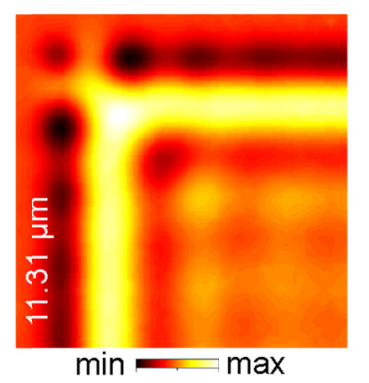

b

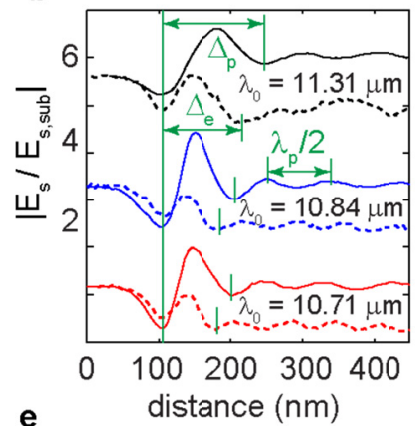

e

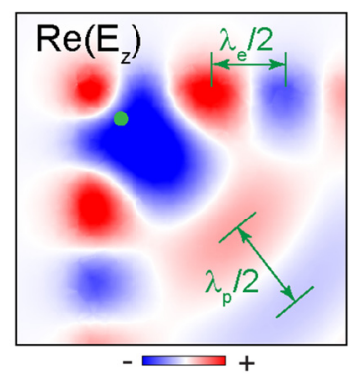

c

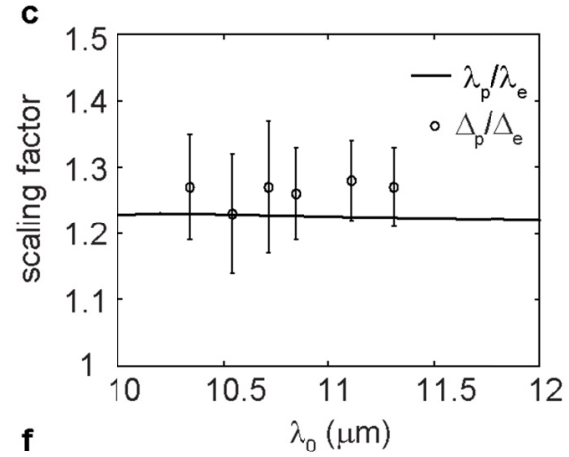

f

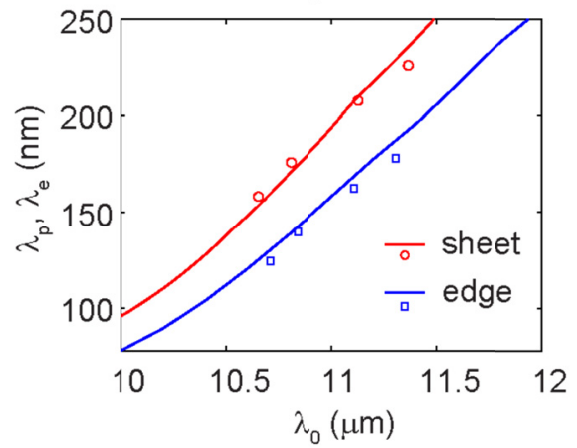

Figure 3: Dispersion of sheet and edge GPs in large graphene structure on $\mathrm{SiO}_{2}$

substrate. a, Experimental near-field image at $\lambda_{0}=11.31 \mu \mathrm{m}$. b, Dashed curves represent individual line profiles along the horizontal white line in a. Solid curves represent averages of 20 individual line profiles in the proximity of the vertical white line in a. c, Averaged ratio $\Delta_{\mathrm{p}} / \Delta_{\mathrm{e}}$ obtained from individual near-field profiles such as the ones shown in b. Error bars show the standard deviation. The line shows the calculated ratio $\lambda_{\mathrm{p}} / \lambda_{\mathrm{e}}$. d, Calculated near-field image. e, Near-field distribution generated by a dipole placed above the graphene rectangle at the position marked by the green dot. f, Dispersion (lines for the theory and symbols for the experiment) of the sheet and edge GPs. Simulations assume $E_{F}=0.33 \mathrm{eV}$ and $\tau=0.1 \mathrm{ps}$. Experimental data for $\lambda_{0}<10.6 \mu \mathrm{m}$ are missing, because $\lambda_{\mathrm{p}}$ could not be measured.

\section{References}

1. Ju, L. et al. Graphene plasmonics for tunable terahertz metamaterials. Nature Nanotechnol. 6, 630-634 (2011).

2. Yan, $H$. et al. Tunable infrared plasmonic devices using graphene/insulator stacks. Nature Nanotechnol. 7, 330-334 (2012).

3. Rodrigo, D. et al. Mid-infrared plasmonic biosensing with graphene. Science 349, 165-168 (2015).

4. Yan, H. et al. Damping pathways of mid-infrared plasmons in graphene nanostructures. Nature Photon. 7, 394-399 (2013). 
5. Tamagnone, M., Fallahi, A., Mosig, J.R. \& Perruisseau-Carrier, J. Fundamental limits and near-optimal design of graphene modulators and non-reciprocal devices. Nature Photon. 8, 556-563 (2014).

6. Fang, Z. et al. Active Tunable Absorption Enhancement with Graphene Nanodisk Arrays. Nano Lett. 14, 299-304 (2014).

7. Fang, Z. et al. Gated Tunability and Hybridization of Localized Plasmons in Nanostructured Graphene. ACS Nano 7, 2388-2395 (2013).

8. Chen, J. et al. Optical nano-imaging of gate-tunable graphene plasmons. Nature 487, 77-81 (2012).

9. Fei, Z. et al. Gate-tuning of graphene plasmons revealed by infrared nanoimaging. Nature 487, 82-85 (2012).

10. Gerber, J.A., Berweger, S., O'Callahan, B.T. \& Raschke, M.B. Phase-Resolved Surface Plasmon Interferometry of Graphene. Phys. Rev. Lett. 113, 055502 (2014).

11. Fei, Z. et al. Edge and Surface Plasmons in Graphene Nanoribbons. Nano Lett. 15, 8271-8276 (2015).

12. Bermudez-Urena, E. et al. Coupling of individual quantum emitters to channel plasmons. Nature Commun. 6 (2015).

13. Wunsch, B., Stauber, T., Sols, F. \& Guinea, F. Dynamical polarization of graphene at finite doping. New J. Phys. 8, 318 (2006).

14. Shung, K.W.K. Dielectric function and plasmon structure of stage-1 intercalated graphite. Phys. Rev. B 34, 979-993 (1986).

15. Hanson, G.W. Dyadic Green's functions and guided surface waves for a surface conductivity model of graphene. J. Appl. Phys. 103, 064302064308 (2008).

16. Vafek, O. Thermoplasma Polariton within Scaling Theory of Single-Layer Graphene. Phys. Rev. Lett. 97, 266406 (2006).

17. Jablan, M., Buljan, H. \& Soljačić, M. Plasmonics in graphene at infrared frequencies. Phys. Rev. B 80, 245435 (2009).

18. Vakil, A. \& Engheta, N. Transformation Optics Using Graphene. Science 332, 1291-1294 (2011).

19. Cai, X. et al. Plasmon-Enhanced Terahertz Photodetection in Graphene. Nano Lett. 15, 4295-4302 (2015).

20. Freitag, M. et al. Photocurrent in graphene harnessed by tunable intrinsic plasmons. Nat Commun. 4 (2013).

21. Koppens, F.H.L. et al. Photodetectors based on graphene, other twodimensional materials and hybrid systems. Nature Nanotechnol. 9, 780793 (2014).

22. Zhu, X. et al. Plasmon-Phonon Coupling in Large-Area Graphene Dot and Antidot Arrays Fabricated by Nanosphere Lithography. Nano Lett. 14, 2907-2913 (2014).

23. Volkov, V.A. \& Mikhailov, S.A. Edge magnetoplasmons: low frequency weakly damped excitations in inhomogeneous two-dimensional electron systems. Sov. Phys. JETP 67, 1639-1653 (1988).

24. Nikitin, A.Y., Guinea, F., García-Vidal, F.J. \& Martín-Moreno, L. Edge and waveguide terahertz surface plasmon modes in graphene microribbons. Phys. Rev. B 84, 161407 (2011).

25. Wang, W., Apell, P. \& Kinaret, J. Edge plasmons in graphene nanostructures. Phys. Rev. B 84, 085423 (2011). 
26. Yan, H. et al. Infrared Spectroscopy of Tunable Dirac Terahertz MagnetoPlasmons in Graphene. Nano Lett. 12, 3766-3771 (2012).

27. Petković, I. et al. Carrier Drift Velocity and Edge Magnetoplasmons in Graphene. Phys. Rev. Lett. 110, 016801 (2013).

28. Schmidt, F.-P. et al. Universal dispersion of surface plasmons in flat nanostructures. Nature Commun. 5 (2014).

29. Ocelic, N., Huber, A. \& Hillenbrand, R. Pseudoheterodyne detection for background-free near-field spectroscopy. Appl. Phys. Lett. 89, 101124 (2006).

30. Miroshnichenko, A.E., Flach, S. \& Kivshar, Y.S. Fano resonances in nanoscale structures. Rev. Mod. Phys. 82, 2257-2298 (2010).

31. Schuller, J.A. et al. Plasmonics for extreme light concentration and manipulation. Nature Mater. 9, 193-204 (2010).

32. Kumar, A. et al. Chiral plasmon in gapped Dirac systems. Phys. Rev. B 93, 041413 (2016).

33. Justin C. W. Song, M.S.R. arXiv:1506.04743 (unpublished)). 


\section{Supplementary Information for "Real-space mapping of tailored sheet and edge plasmons in graphene nanoresonators"}

\section{A. Numerical simulations}

\section{A.1. Description of the model}

In the s-SNOM experiments the sharp metallic tip is illuminated by the incident laser beam. The tip acts as an optical antenna converting the incident light into a strongly confined near field below the tip apex. This localized near field provides the necessary momentum to excite plasmons in the graphene nanoresonators. Both numerical ${ }^{1,2}$ and analytical ${ }^{1}$ quantitative studies of s-SNOM experiments meet substantial difficulties in simulating near-field images, owing to the complex near-field interaction between the tip and the sample. For example, numerically calculated s-SNOM images of plasmons in graphene have not been able up to now to explain details observed in experimental s-SNOM images. Among others, it has been not understood up to now why graphene edges appear dark in experimental s-SNOM images.

In order to achieve a deeper understanding of s-SNOM images of graphene nanoresonators, we developed a simple model that allows for elucidating the physics behind the s-SNOM images. We approximate the tip by a dipole source (with a constant dipole moment). This is different from the usual dipole model, in which the effective dipole moment is given by the product of the exciting electric field and the polarizability of a sphere ${ }^{3}$. Our significant simplification of the usual dipole model can be justified by the fact that the volume of the real metallic tip is approximately 5 orders of magnitude larger than the volume of the graphene resonators $\left(20 \mu \mathrm{m}^{3}\right.$ for a pyramid-shape tip of $10 \mu \mathrm{m}$ length against $3 \cdot 10^{-4} \mu \mathrm{m}^{3}$ for a square graphene resonator with a side length of $1 \mu \mathrm{m}$ ). Because of such a mismatch in volumes, the polarizability of the dipole is weakly affected by the plasmon excited in the graphene nanoresonators. Back-action of the graphene plasmons (GPs) onto the tip can be thus neglected.

When the point dipole source is placed above the substrate, it generates the near field in a confined volume around the dipole. The field above the substrate, $\mathrm{E}_{z, \text { sub }}$, can be represented by the sum of the primary near field (generated by the dipole without the substrate) and the near field reflected from the substrate ${ }^{4}$. When a graphene nanoresonator is placed below the dipole on the substrate, the near field $E_{z, \text { sub }}$ couples to graphene plasmons, the later described by their electric field $E_{z, G P}$. The total near field $E_{z}$ between the dipole and the resonator can be approximated by sum of $\mathrm{E}_{z, \text { sub }}$ and $\mathrm{E}_{\mathrm{z}, \mathrm{GP}}$ (the latter being a function of $\mathrm{E}_{z, \mathrm{sub}}$ ), $\mathrm{E}_{\mathrm{z}}=\mathrm{E}_{\mathrm{z}, \mathrm{sub}}+$ $\mathrm{E}_{\mathrm{z}, \mathrm{GP}}\left(\mathrm{E}_{z, \mathrm{sub}}\right)$. Calculating the amplitude of the near field, $\left|\mathrm{E}_{\mathrm{z}}\right|$, as a function of the dipole position (x,y), (for the full wave simulations we use Comsol software) we can simulate nearfield images. Even the finest details in the experimental s-SNOM images are well reproduced by these simulated images (see Fig. 1b,d, Fig. 2 and Fig. 3 a,d), which lets us conclude that the calculated field between the dipole and graphene resonator, $\mathrm{E}_{\mathrm{z}}$, provides a valid numerical description of the signals measured by s-SNOM.

We stress that the simplicity of our model and its capability to reproduce, for the first time, even the finest details of the experimental near-field images are the key to identifying puzzling negative image contrast as Fano-like interference and to disentangling of the various overlapping GP modes. 
We note that our simulations (where fields are calculated) are significantly different to maps of the local density of the optical states (LDOS) ${ }^{5}$, which were previously used to describe GP patterns in near-field images. Most important, a detailed comparison of LDOS maps and experimental near-field images (e.g. in $^{5}$ ) shows that LDOS maps cannot fully reproduce and explain the experimental near-field images (for example why graphene edges appear dark in near-field images).

\section{A.2. Modes in the disk resonators}

Fig. 1S shows the numerical spectral analysis of the plasmonic modes in the disk resonators. The vertical point dipole source was placed at the distance $\mathrm{h}=140 \mathrm{~nm}$ from the disk surface either above the centre or the edge (marked by the points A and B in Fig. S1a, respectively). We calculated the near-field $\mathrm{E}_{\mathrm{z}}$, at $\mathrm{Z}_{\mathrm{NF}}=15 \mathrm{~nm}$ above the disk (illustrated in Fig. S1a) as a function of the disk diameter.

The upper panel of Fig S1b, shows the absolute value of the total near-field, $\left|E_{z}\right|$, for convenience normalized to $\left|\mathrm{E}_{\mathrm{z}, \mathrm{sub}}\right|$ (the same result is shown in Fig. 1i,j for $\lambda_{0}=11.31 \mu \mathrm{m}$ ). We see the asymmetric resonance peaks, which, as explained in the main text, correspond to the excitation of various GP modes. The asymmetry of the resonances can be explained by a Fano-like interference ${ }^{6}$, in which a non-resonant process (near-field created by the dipole $E_{z, \text { sub }}$ ) interferes with the resonant one (near-field of the resonantly excited GP mode $\left.\mathrm{E}_{\mathrm{z}, \mathrm{GP}}\left(\mathrm{E}_{\mathrm{z}, \mathrm{sub}}\right)\right)$. This interference can be described by $\mathrm{E}_{\mathrm{z}}=\mathrm{E}_{\mathrm{z}, \mathrm{sub}}+\mathrm{E}_{\mathrm{z}, \mathrm{GP}}\left(\mathrm{E}_{\mathrm{z}, \mathrm{sub}}\right)$, as explained above in Section A.1. We can thus calculate the GP field by a complex-valued subtraction: $\mathrm{E}_{z, \mathrm{GP}}=\mathrm{E}_{\mathrm{z}}-\mathrm{E}_{\mathrm{z}, \mathrm{sub}}$. The amplitude and phase spectra of the resulting plasmon field, $\mathrm{E}_{z, \mathrm{GP}}$, are shown in the middle and lower panels of Figs. S1b. In the amplitude spectra, the resonance peaks are nearly symmetric and accompanied by strong phase changes (which is a typical observation for resonance phenomena). The remaining slight asymmetry of the peaks in the amplitude spectra we attribute mainly to the overlap of the different excited GP modes. We find that the peak positions of $\mathrm{E}_{\mathrm{z}, \mathrm{GP}}$ (Fig. S1b, middle panel) are shifted to larger disk diameters compared to the peak positions observed in the total field $E_{z}$ (Fig. S1b, upper panel). Importantly, it is the peak position of $E_{z, G P}$ that determines the resonance of the GP modes, rather than the peak position of $E_{z}$. However, the near-field distributions calculated either at the peak position of $E_{z}$ or $E_{z, G P}$ do not have any significant visual difference. Therefore, the GP modes shown in Fig. If of the main text were calculated at the peak positions found in the spectra $\mathrm{E}_{\mathrm{z}}$. 

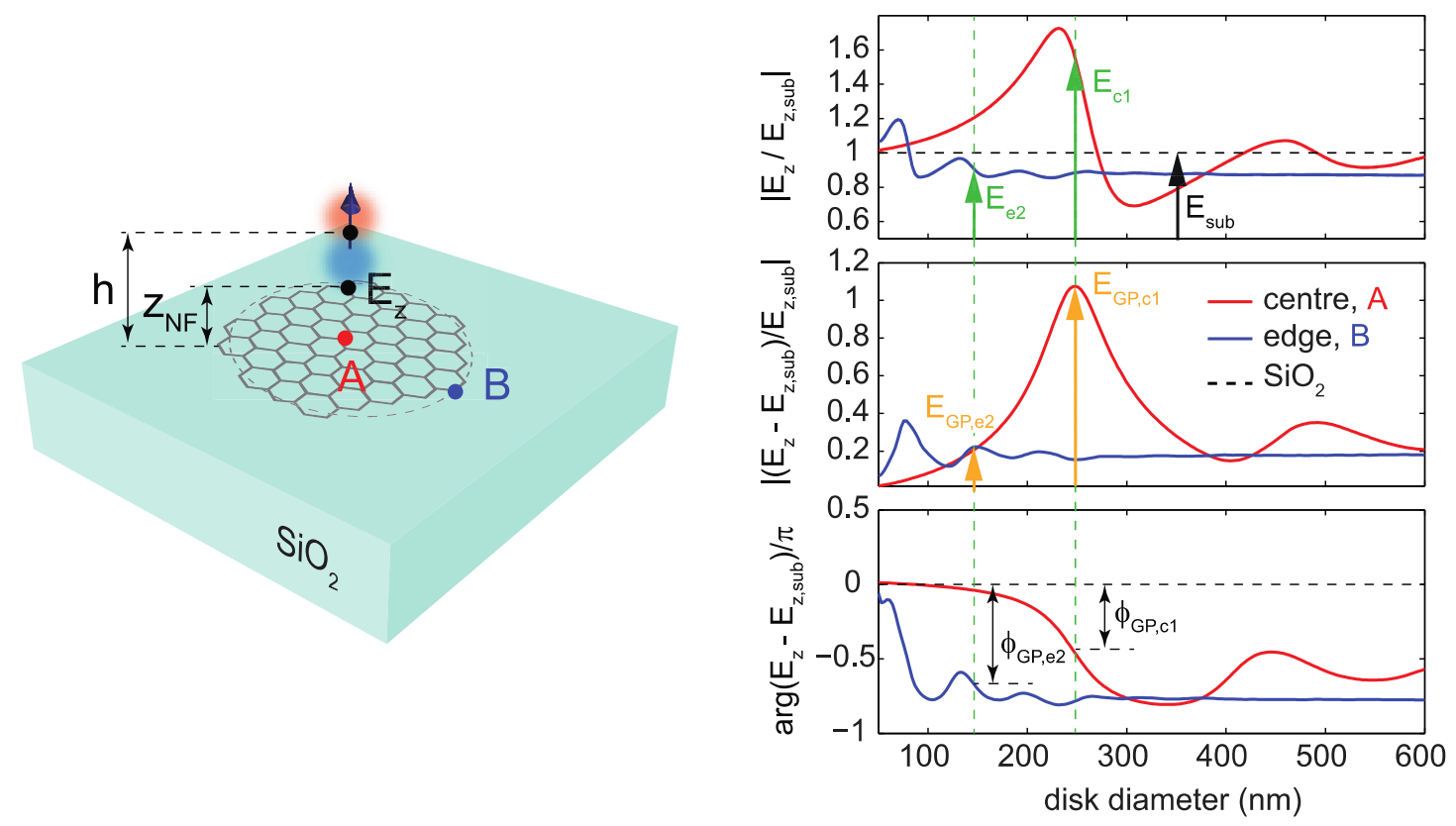

Figure S1: Extracting GP modes in graphene disk nanoresonators from the near-filed spectra. a, schematics of the studied structure: graphene disk on $\mathrm{SiO}_{2}$ substrate and the vertical point dipole above. The dipole-resonator separation is $\mathrm{h}=140 \mathrm{~nm}$, the near-field is recorded at the point $\mathrm{z}_{\mathrm{NF}}=15 \mathrm{~nm}$. $\mathbf{b}$, the near-field (top), modified nearfield (middle) and the phase of the GP mode near-field (bottom) as a function of the disk diameter. The vertical green lines mark the position of the dipolar resonance 2 and the first-order breathing mode $\mathrm{c} 1$ (see the near-field distributions of the modes in Fig. 1f). Simulations assume a Fermi energy $E_{F}=0.33 \mathrm{eV}$, a relaxation time $\tau=0.1$ ps, and the wavelength $\lambda_{0}=11.31 \mu \mathrm{m}$. The vertical arrows in the top and middle panels of $\mathbf{b}$ approximately correspond to those shown in the vector diagram of Fig. 1e in the main text. The double-end arrows shown in the bottom panel of $\mathbf{b}$ indicate the phase shifts of the GP modes $\mathrm{c} 1$ and $\mathrm{e} 2$.

Based on the results shown in Fig. S1b, we can explain the positive and negative image contrasts of GPs. To that end, we plot the different fields (qualitatively) in the complex plane shown in Fig. 1e of the main text. The amplitude and phase of the GP fields $\mathrm{E}_{\mathrm{GP}, \mathrm{cl}}$ and $\mathrm{E}_{\mathrm{GP}, \mathrm{e} 1}$ (orange arrows in Fig. 1e) correspond to the orange arrows in Fig. S1b (middle panel), respectively black double-head arrows in Fig. S1b (lower panel). The GP fields $\mathrm{E}_{\mathrm{GP}, \mathrm{cl}}$ and $\mathrm{E}_{\mathrm{GP}, \mathrm{el}}$ (orange arrows in Fig. 1e) add to the field $\mathrm{E}_{\text {sub }}$ below the dipole (black arrows in Fig. 1e and dashed black line in Fig. S1b (upper panel)), yielding the total fields $\mathrm{E}_{\mathrm{c} 1}$ and $\mathrm{E}_{\mathrm{e} 2}$ (green arrows in Fig. 1e and Fig. S1b (upper panel). Because the phase difference $\varphi_{\mathrm{c} 1}$ between $\mathrm{E}_{\mathrm{GP}, \mathrm{c} 1}$ and $E_{\text {sub }}$ is smaller than $\pi / 2$, the total field amplitude $\left|E_{c 1}\right|$ is increased (leading to positive contrast of the centre of the $250 \mathrm{~nm}$ diameter disks in Figs. 1b,d). On the contrary, because the phase difference $\varphi_{\mathrm{e} 2}$ between $\mathrm{E}_{\mathrm{GP}, \mathrm{e} 2}$ and $\mathrm{E}_{\text {sub }}$ is larger than $\pi / 2$, the total field amplitude $\left|\mathrm{E}_{\mathrm{e} 2}\right|$ is decreased (leading to negative contrast of the edges of the $125 \mathrm{~nm}$ diameter disks in Figs. 1b,d).

\section{A.3. Modes in the rectangular resonators}

In Fig. S2, analogously to the graphene disk resonators, we performed a similar analysis of the modes in the graphene rectangles (illustrated in Fig. S2a). We calculated the near fields $E_{z}$ and $\mathrm{E}_{\mathrm{z}, \mathrm{GP}}$ as a function of the excitation wavelength, $\lambda_{0}$, for different positions of the point dipole. The top panels of Figs. S2b-d display the spectra of the total field $E_{z}$ (the same graphs are shown in Fig. 2 of the main text). As described above, we subtract the dipole field, $E_{z, \text { sub }}$, from the total field, $E_{z}$, in order to obtain the GP field, $E_{z, G P}$. Amplitude and phase of $E_{z, G P}$ are shown in the middle and lower panels of Figs. S2b-d. As in Fig. 1Sb, we observe nearly 
symmetric peaks in the amplitude spectra, which are accompanied by strong phase changes. We find that the peak positions of $\mathrm{E}_{\mathrm{z}, \mathrm{GP}}$ (Figs. S1b-d, middle panel) are shifted to smaller wavelengths compared to the peak positions observed in the total field $\mathrm{E}_{\mathrm{z}}$ (Figs. S1b-d, upper panel). For that reason, the GP modes in the main text were calculated at the peak positions found in the spectra $E_{z, G P}$. In the spectra shown in Fig. 2 of the main text (displaying $E_{z}$ ), the mode positions (marked s1, e1 etc) thus do not coincide with the peak positions.

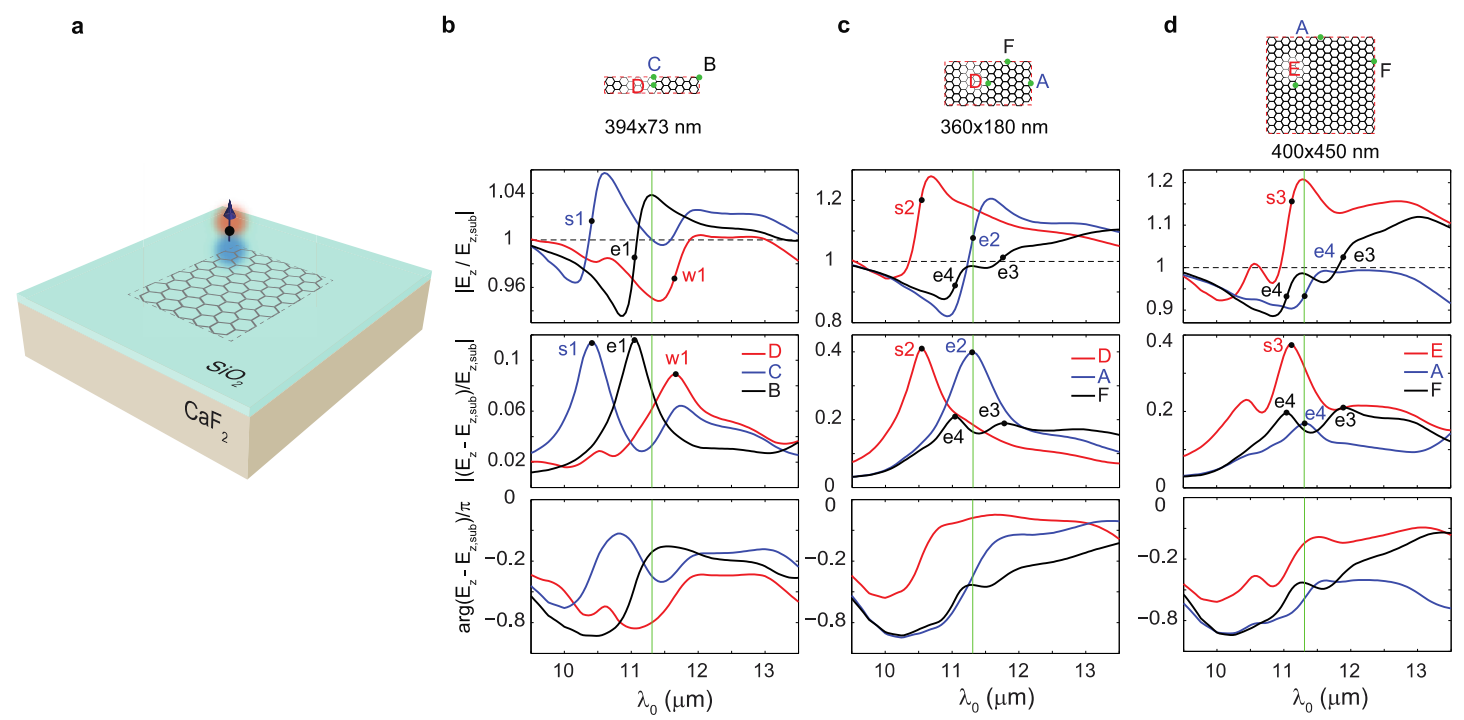

Figure S2: Extracting GP modes in graphene rectangular nanoresonators from the near-filed wavelength spectra. a, schematics of the studied structure: graphene rectangle on 5-nm thick $\mathrm{SiO}_{2}$ film placed on $\mathrm{CaF}_{2}$ substrate and the vertical point dipole above. The dipole-resonator separation is $\mathrm{h}=120 \mathrm{~nm}$, the near-field is recorded at the point $\mathrm{Z}_{\mathrm{NF}}=35 \mathrm{~nm}$. b, the near-field (top), modified near-field (middle) and the phase of the modified near-field (bottom) as a function of the wavelength, $\lambda_{0}$. The vertical green lines mark the position of the imaging wavelength $\lambda_{0}=11.31 \mu \mathrm{m}$ for the near-field images and near-field distributions in Fig. $2 \mathbf{a}-\mathbf{c}$ of the main text. Simulations assume Fermi energies $E_{F}=0.30 \mathrm{eV}$ in $\mathbf{a}, \mathrm{E}_{\mathrm{F}}=0.28 \mathrm{eV}$ in $\mathbf{c}$ and $\mathrm{E}_{\mathrm{F}}=0.31 \mathrm{eV}$ in d, and a relaxation time $\tau=0.1 \mathrm{ps}$.

\section{B. Reflection of edge modes at defects}

In the near-field images of Fig. 2a-c of the main text we observe black dots decorating the edges (modulation of the contrast along the edges) of the graphene nanoresonators. We explain them as edge GP modes, which due to reflection at the corners yield standing wave patterns, i.e. Fabry-Perot resonances. The excellent agreement between the experimental and simulated near-filed images, as well as the numerical spectral analysis (shown in Fig. 2d-f and Fig. S2b-d) corroborate this explanation.

We note that the black dots are always present - and with well-defined circular shape - at the corners of the rectangles. For the large rectangle in Fig 2c, however, we observe that the black dots along the sides are less regular and less defined. We attribute this observation to defects at the edges acting as additional reflectors for edge $\mathrm{GP}^{7}$. The multiple (weaker) reflections of GPs at defects between the corners thus result in less regular dot patterns along the edges. 

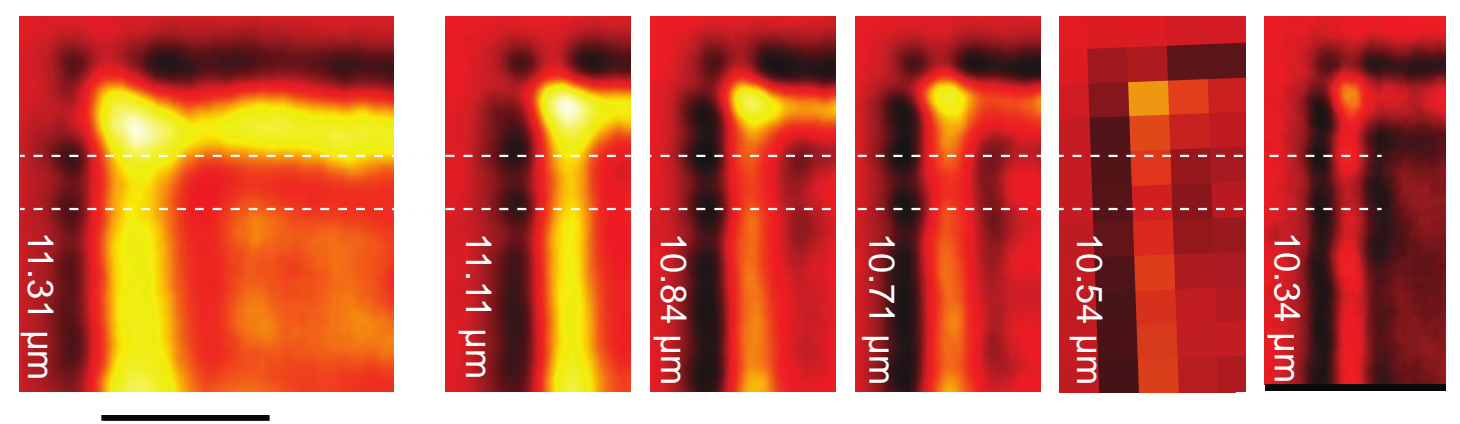

$200 \mathrm{~nm}$

Figure S3: Near-field images of the large graphene structure of Fig. 3a, recorded at different illumination wavelengths (indicated in the images).

Our assumption that edge GPs are reflected at edge defects is confirmed by several experiments (not shown), where we observe black dots far away from the corner (further than the propagation distance of the edge GPs). The periodicity of these dot patterns does not change upon variation of the illumination wavelength, indicating that the dot pattern is not due to interference of propagating edge plasmons but rather due to reflection of the edge plasmons at stationary defect. The centre of the dot marks the location where the reflection occurs. A similar observation is actually made at the vertical edge of Fig. 3. Imaging at different wavelengths (Fig. S3) reveals that the position and periodicity of some of the black dots along the vertical edge does not change (marked by horizontal dashed lines in Fig. S3). We conclude that the dots along the vertical edge are caused by interference of tip-launched edge plasmons being reflected not only at the corner but also at defects along the edge. Consequently, the periodicity of the black dots is determined by the distribution of defects rather than by the plasmon wavelength, preventing us to determine the dispersion form measuring the periodicity of the black dots. On the other hand, the plasmonic origin of the black dots at edges and corners is confirmed by their increasing diameter with increasing illumination wavelength.

Along the horizontal graphene edge of Fig. 3 we find that the intensity of the black dots (and subsequently the presence of defects) is rather weak (i.e. the graphene edge is seen as a nearly homogeneously dark fringe). Only at the corner we observe a well-defined black spot. We conclude that edge plasmons are dominantly reflected at the corner. The absence of black dots further away from the corner we attribute to the short propagation length of the edge GPs, owing to their strong confinement and rather ill-defined edge geometry (note that the edge were produced by etching), the later resulting in increased damping. Because of the dominating reflection of edge plasmons at the corner, we succeeded to determine the dispersion of edge plasmons by analyzing near-field profiles along the horizontal edge (see description of Fig. 3 in the main text).

\section{Notes for the fabrication of the graphene resonators}

$\mathrm{SiO}_{2}$ is the typical substrate for graphene device fabrication. Because of its phonons and rather large refractive index in the mid-infrared spectral range, plasmons in graphene could exhibit additional damping and screening. For that reason, we also used the low-refractive index material $\mathrm{CaF}_{2}$ as substrate for fabrication of graphene nanoresonators. Additionally, $\mathrm{CaF}_{2}$ does not exhibit phonon resonances in the spectral range considered in our work. We found, however, that chemical doping of graphene by the $\mathrm{CaF}_{2}$ substrate was weak and did not yield strong plasmon excitation. For that reason, we added a few nanometer thick layer of $\mathrm{SiO}_{2}$ onto the $\mathrm{CaF}_{2}$ substrate in order to enhance the chemical doping of the graphene. While on such composite substrates we clearly observe graphene plasmons, their strength and 
quality is similar compared to graphene plasmons on standard $\mathrm{SiO}_{2}$ substrates. In this work we present the best-quality results, which for disk resonators were obtained on a standard $\mathrm{SiO}_{2}$ substrate (Fig. 1), for rectangular resonators on a $\mathrm{CaF}_{2}$ substrate with thin $\mathrm{SiO}_{2}$ layer (Fig. 2), and for graphene plasmon edge modes on a standard $\mathrm{SiO}_{2}$ substrate (Fig. 3).

\section{References for Supplementary Information:}

1. McLeod, A.S. et al. Model for quantitative tip-enhanced spectroscopy and the extraction of nanoscale-resolved optical constants. Phys. Rev. B 90, 085136 (2014).

2. Chen, J. et al. Strong Plasmon Reflection at Nanometer-Size Gaps in Monolayer Graphene on SiC. Nano Lett. 13, 6210-6215 (2013).

3. Hillenbrand, R. \& Keilmann, F. Complex Optical Constants on a Subwavelength Scale. Phys. Rev. Lett. 85, 3029-3032 (2000).

4. Novotny, L. \& Hecht, B. Principles of Nano-Optics. (Cambridge University Press, 2006).

5. Chen, J. et al. Optical nano-imaging of gate-tunable graphene plasmons. Nature 487, 77-81 (2012).

6. Miroshnichenko, A.E., Flach, S. \& Kivshar, Y.S. Fano resonances in nanoscale structures. Rev. Mod. Phys. 82, 2257-2298 (2010).

7. Fei, Z. et al. Edge and Surface Plasmons in Graphene Nanoribbons. Nano Lett. 15, 8271-8276 (2015). 